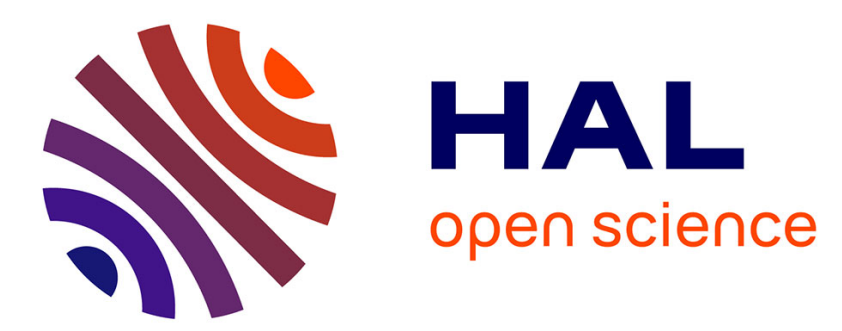

\title{
EXCITATION COLLISIONNELLE DES ATOMES ET DES MOLÉCULES DANS LE MILIEU INTERSTELLAIRE (ASPECT THÉORIQUE)
}

Jean-Michel Launay

\section{- To cite this version:}

Jean-Michel Launay. EXCITATION COLLISIONNELLE DES ATOMES ET DES MOLÉCULES DANS LE MILIEU INTERSTELLAIRE (ASPECT THÉORIQUE). Journal de Physique Colloques, 1978, 39 (C1), pp.C1-43-C1-44. 10.1051/jphyscol:1978107 . jpa-00217303

\section{HAL Id: jpa-00217303 https://hal.science/jpa-00217303}

Submitted on 1 Jan 1978

HAL is a multi-disciplinary open access archive for the deposit and dissemination of scientific research documents, whether they are published or not. The documents may come from teaching and research institutions in France or abroad, or from public or private research centers.
L'archive ouverte pluridisciplinaire HAL, est destinée au dépôt et à la diffusion de documents scientifiques de niveau recherche, publiés ou non, émanant des établissements d'enseignement et de recherche français ou étrangers, des laboratoires publics ou privés. 


\title{
EXCITATION COLLISIONNELLE DES ATOMES ET DES MOLÉCULES DANS LE MILIEU INTERSTELLAIRE (ASPECT THÉORIQUE)
}

\author{
J.-M. LAUNAY \\ Département d'Astrophysique Fondamentale, Observatoire de Meudon, 92190 Meudon, France
}

\begin{abstract}
Résumé. - Récemment, des progrès ont été accomplis dans la détermination théorique de l'excitation collisionnelle des transitions de structure fine et des transitions de rotation pour les molécules d'intérêt astrophysique. Les surfaces d'énergie potentielle des systèmes comportant peu d'électrons sont accessibles aux méthodes $a b$ initio et le calcul de la collision peut être envisagé par les méthodes quantiques, pour des énergies basses et des systèmes légers.
\end{abstract}

\begin{abstract}
Recently, progress have been made in the theoretical determination of fine structure transitions and in rotational transitions by collisions in molecules of astrophysical interest. Potential energy surfaces may be computed by ab initio techniques in few-electron systems and dynamical calculations may be done by quantum methods for low energies and light systems.
\end{abstract}

La découverte depuis une dizaine d'années de molécules dans le milieu interstellaire par les radioastronomes a conduit un certain nombre de chercheurs à se pencher sur la physique des collisions entre particules lourdes à basse énergie cinétique

$$
\left(10^{-3} \mathrm{eV} \lesssim E \lesssim 10^{-1} \mathrm{eV}\right) \text {. }
$$

En effet, la connaissance des sections efficaces d'excitation collisionnelle des molécules ou des atomes ainsi que des probabilités de transition radiative est nécessaire pour expliquer :

a. - L'intensité des raies d'émission provenant des molécules comme $\mathrm{OH}, \mathrm{CO}, \mathrm{HCN}, \ldots$ (transitions entre niveaux de rotations, transitions entre doublets $\Lambda$, transitions entre niveaux hyperfins) qui se trouvent dans le domaine des ondes radios, et des atomes comme $\mathrm{C}, \mathrm{C}^{+}, \mathrm{O}$ (transitions de structure fine) qui se trouvent dans l'infra-rouge lointain.

b. - Le refroidissement du milieu interstellaire qui est dû aux molécules $\mathrm{CO}$ dans les milieux froids $\left(T_{k} \sim 10 \mathrm{~K}\right)$, aux atomes $\mathrm{C}, \mathrm{C}^{+}, \mathrm{O}$ dans les milieux plus chauds et moins denses [1].

L'excitation de ces transitions est due aux collisions avec les espèces atomiques ou moléculaires les plus abondantes dans le milieu interstellaire qui sont $\mathbf{H}_{2}$ dans les milieux à forte densité, $\mathbf{H}$ quand le milieu est moins dense, ou les ions dans le cas des molécules polaires. Le calcul théorique des collisions intermoléculaires se scinde en deux problèmes distincts : d'une part, la détermination des courbes ou surfaces de potentiel d'interaction entre les deux particules, d'autre part, la détermination de la matrice $S$ de collision qui est l'amplitude de probabilité de trouver les états finaux correspondants à un état initial donné.
1. Détermination du potentiel d'interaction. - Nous considérerons uniquement les méthodes dites $a b$ initio, c'est-à-dire ne faisant pas intervenir de paramètres ajustables.

- La méthode de Hartree-Fock suppose que chaque électron se déplace dans le champ moyen créé par les autres électrons ; elle ne permet pas d'obtenir l'énergie de dispersion qui est due à la corrélation entre les positions des électrons de chaque système et qui provoque un minimum de potentiel de l'ordre de $1 / 100 \mathrm{eV}$ (minimum de Van der Waals) dans les interactions entre systèmes à couches fermées. Elle peut cependant être utilisée aux faibles distances intermoléculaires, dans la partie répulsive, comme dans le cas $\mathrm{H}-\mathrm{CO}$ [2].

- La méthode d'interaction de configuration converge vers l'énergie exacte quand la base des fonctions d'ondes est augmentée; elle permet d'obtenir l'énergie de dispersion mais demande des moyens de calcul environ un ordre de grandeur supérieurs à ceux de la méthode de Hartree-Fock. Elle a été utilisée dans le cas des interactions $\mathrm{C}^{+}-\mathrm{H}_{2}$ [3], $\mathrm{C}^{+}-\mathrm{H}$ [4], $\mathrm{He}-\mathrm{H}_{2} \mathrm{CO}$ [5].

Ces deux méthodes souffrent du défaut que l'énergie d'interaction qui est une toute petite partie de l'énergie totale s'obtient par différence entre l'énergie des systèmes en interaction et l'énergie des systèmes isolés ; aux grandes distances, des erreurs de cancellation peuvent donc se manifester.

Aussi, plusieurs méthodes permettant d'obtenir directement l'énergie d'interaction ont été utilisées :

- La méthode du gaz d'électrons de Gordon et $\mathrm{Kim}$ [26] sur les couples $\mathrm{CO}-\mathrm{He}$ [2], $\mathrm{HCN}-\mathrm{He}$ [6], $\mathrm{H}_{2} \mathrm{CO}-\mathrm{He}$ [7], $\mathrm{HCO}^{+}-\mathrm{He}$ [8], $\mathrm{N}_{2} \mathrm{H}^{+}-\mathrm{He}$ [8], $\mathrm{NH}_{3}-\mathrm{He}$ [9]. Elle est restreinte aux systèmes à couches fermées et sa validité est en cours de discussion. 
- Une méthode de perturbation avec échange sur les couples $\mathrm{C}-\mathrm{H}, \mathrm{O}-\mathrm{H}, \mathrm{C}^{+}-\mathrm{H}$ aux grandes distances internucléaires [10].

- Une méthode développée par E. Kochanski (Laboratoire de Chimie Quantique-Strasbourg) qui consiste à calculer l'énergie de dispersion par une méthode perturbative. Cette méthode, valable uniquement pour les systèmes à couches fermées, est actuellement utilisée sur le couple $\mathrm{CO}-\mathrm{H}_{2}$ [11].

Enfin, aux grandes distances, on utilise le développement en $1 / R$ du potentiel d'interaction qui est donné sous la forme d'une somme de trois termes: énergie d'interaction électrostatique, énergie d'induction, énergie de dispersion [12].

2. Calcul de la matrice $S$ de collision. - Grâce aux développements récents de la technologie des ordinateurs, la résolution exacte des équations quantiques couplées de la diffusion a pu être entreprise depuis 1970 environ.

La fonction d'onde totale du système est projetée sur une hase de fonctions d'ondes des systèmes isolés [13] ; l'approximation nécessaire est la troncature de la base de façon à n'avoir qu'un nombre fini d'équations couplées qui peut aller, à l'heure actuelle, jusqu'à une centaine. Cette méthode a l'avantage d'assurer la conservation de l'énergie et du moment angulaire totaux du système et est donc, en général, préférable aux méthodes semi-classiques.
Elle a été employée dans de nombreux cas où l'un des partenaires au moins est un atome : $\mathrm{CO}-\mathrm{He}[2$, 16], $\mathrm{HCN}-\mathrm{He}[6], \mathrm{H}_{2} \mathrm{CO}-\mathrm{He}[21], \mathrm{HCO}^{+}-\mathrm{He}[8]$, $\mathrm{N}_{2} \mathrm{H}^{+}-\mathrm{He}$ [8], $\mathrm{NH}_{3}-\mathrm{He}$ [9], $\mathrm{CO}-\mathrm{H}[2,22], \mathrm{C}^{+}-\mathrm{H}[23$, 24], C-H [25], O-H [25].

Elle ne convient pas quand de nombreux états sont fortement couplés, comme dans le cas de molécules à grand moment d'inertie.

Une méthode approximative, basée sur la conservation approchée de la projection du moment cinétique total sur l'axe interparticule a été développée par McGuire et Kouri [14]. Elle conduit à un découplage partiel des équations et donne de bons résultats quand les forces à longue portée ne sont pas prépondérantes; elle a été testée, par exemple, sur les systèmes $\mathrm{CO}-\mathrm{He}$ $[15,16]$ et $\mathrm{HCN}-\mathrm{He}[17]$.

La méthode quantique est présentement étendue aux collisions avec l'hydrogène moléculaire; les collisions $\mathrm{H}_{2}-\mathrm{H}_{2}$ [18], $\mathrm{C}^{+}-\mathrm{H}_{2}$ [19] ont été traitées et la collision $\mathrm{CO}-\mathrm{H}_{2}$ est en cours d'étude à la fois sur le plan théorique [20] et sur le plan expérimental par une technique de double résonance (voir l'exposé de Philippe Brechignac).

Il est en effet utile de mesurer l'importance de la structure rotationnelle de $\mathrm{H}_{2}$ dans les calculs, le nombre de points à calculer pour avoir une bonne représentation de la surface de potentiel étant très important.

\section{Bibliographie}

[1] Dalgarno, A. et MC Cray, R. A., Annu. Rev. Astron. Astrophys. 10 (1972) 375.

[2] Green, S. et Thaddeus, P., Astrophys. J. 205 (1976) 766.

[3] Liskow, D. H., Bender, C. F. et Schaeffer, H. F., $J$. Chem. Phys. 61 (1974) 2507.

[4] Green, S., Bagus, P. S., Liu, B., Mc Lean, A. D. et Yoshimine, M., Phys. Rev. A 5 (1972) 1614

[5] Garrison, B. J., Lester, W. A., Sieghahn, P. et SchaeFER, H. F., Chem. Phys. 63 (1976) 1449.

[6] Green, S. et Thaddeus, P., Astrophys. J. 191 (1974) 653.

[7] Green, S., Garrison, B. J. et Lester, W. A., J. Chem. Phys. 63 (1975) 1154

[8] GreEn, S., Astrophys. J. 201 (1975) 366.

[9] Green, S., J. Chem. Phys. 64 (1976) 3463.

[10] Bazet, J. F., Harel, C., Mc Carroll, R. et Riera, A., Astron. Astrophys. 43 (1975) 223

[11] Kochanski, E., Prissette, J. et Flower, D., 1978 (à paraître dans Chem. Phys.).

[12] Buckingham, A. D., Intermolecular Forces, édité par J. O. HirsChFELdER (New York : Wiley) 1967, p. 107.
[13] Arthurs, A. M. et Dalgarno, A., Proc. R. Soc. A 256 (1960) 540.

[14] Mc Guire, P. et KourI, D. J., J. Chem. Phys. 60 (1974) 2488.

[15] Green, S., Chem. Phys. Lett. 38 (1976) 293.

[16] Launay, J. M., J. Phys. B 10 (1976) 1823.

[17] MC Guire, P., Chem. Phys. 13 (1976) 81.

[18] Green, S., J. Chem. Phys. 62 (1975) 2271.

[19] Flower, D. et LAUNAY, J. M., J. Phys. B., 1978 (à paraître).

[20] Flower, D. et Launay, J. M., J. Phys. B., 1978 (à paraître).

[21] Garrison, B. J., Lester, W. A. et Miller, W. H., $J$. Chem. Phys. 65 (1976) 2193.

[22] Chu Shit-I et Dalgarno, A., Proc. R. Soc. A 342 (1975) 191.

[23] Weisheit, J. C. et Lane, N. F., Phys. Rev. A 4 (1971) 171.

[24] Launay, J. M. et Rouefe, E., J. Phys. B 10 (1977) 879.

[25] Launay, J. M. et Rouefr, E., Astron. Astrophys. 36 (1977) 289.

[26] Gordon, R. G. et KIM, Y. S., J. Chem. Phys. 56 (1972) 3122 